\title{
Intra-Car Multi-hop Wireless Sensor Networking: A Case Study
}

\author{
Morteza Hashemi*, Wei Si*, Moshe Laifenfeld ${ }^{\dagger}$, David Starobinski* and Ari Trachtenberg* \\ * Department of Electrical and Computer Engineering, Boston University, USA \\ \{mhashemi, weisi, staro, trachten\}@bu.edu \\ †Wireless Enablers Group, GM Advanced Technical Center, Israel \\ moshe.laifenfeld@gm.com
}

\begin{abstract}
Modern vehicles incorporate dozens of sensors to provide vital sensor data to the electronic control units (ECUs), typically through physical wires, which increase the weight, maintenance, and cost of cars. Wireless sensor networks (WSN) have been contemplated for replacing the current physical wires with wireless links, although existing networks are all single hop, presumably because cars are small enough to be covered with lowpower communication, and multi-hop networking requires organizational overhead. In contradistinction with previous works, we experimentally investigate the usage of multi-hop wireless communication to support intra-car sensor networking. Extensive tests, run under various vehicular environments, indicate the potential for significant reliability, robustness, and energy-usage improvements over existing single-hop approaches. Our implementation is based on the Collection Tree Protocol (CTP), a state-of-the-art multi-hop data collection protocol.
\end{abstract}

\section{INTRODUCTION}

Wireless sensor networks (WSN) boast numerous applications ranging from home appliances control, to environmental monitoring, and smart healthcare. More recently, they have also demonstrated benefits for intelligent transport systems: monitoring aircraft systems and parameters [1], monitoring wheel bearings on trains [2], and connecting sensors, switches and actuators inside cars [3-5]. In these applications, the ability to reliably aggregate data in one or several processing centers is critical to the monitoring capabilities of the sensors, which are typically constrained in both energy and computational power. For transport systems, this aggregation is further complicated by the dynamic channel properties that vehicles may experience as they travel through areas with different radio interference patterns or road quality.

To date, several single-hop communication models based on Zigbee, RFID, and ultra-wideband technologies, have been examined for intra-car wireless networking [3-5]; in these networks, all sensor nodes communicate in a point-to-point fashion (within a star topology) with the central node. For largescale deployments, especially over large physical distances, it may be advantageous to arrange sensors in a multi-hop network, with some sensors relaying information from other sensors onto the central node (or collection root). Within vehicles, however, it is not clear whether multi-hop networking is worthwhile, as the small distances involved typically allow sensors to reach the central node using low or medium transmission power.
Indeed, multi-hop networking adds communication overhead to the system, requiring exchange of metadata (e.g., topology information) and utilizing available bandwidth for packet relaying (with some incident interference to other sensors).

In this work, we show that, notwithstanding the caveats mentioned above, multi-hop networking may provide clear benefits within cars. Specifically, despite its greater overhead, it can (a) enhance system reliability, (b) provide robust performance, and (c) reduce communication energy. We utilize the Collection Tree Protocol (CTP) [6] as a multi-hop network layer protocol, as it is (i) widely deployed, (ii) well researched, and (iii) the basis of the IPv6 Routing Protocol for Low-power and Lossy Networks (RPL) standard [7]. Our results show, for example, that the packet delivery rate of a node using a single-hop topology protocol can be below $80 \%$ in practical scenarios, whereas CTP improves reliability performance beyond $95 \%$ across all nodes while simultaneously reducing radio energy consumption.

We perform several experiments to explore the effects of environmental conditions on the performance of intra-car WSN; for instance, our results show that engine noise does not have much effect on the performance, even though it adds $2-4 \mathrm{~dB}$ in the noise power [3]. On the other hand, CTP achieves clearly better performance when a vehicle is parked in a covered area (i.e., garage) than in an open area outdoor parking. To the best of our knowledge, this is the first demonstration of the significant benefits possible from multi-hop networking within vehicles. A preliminary version of our results was presented in [8].

The rest of this article is organized as follows. In Section II we provide background on existing intra-car WSN models, and on the CTP protocol. Our experimental setup is described in Section III, followed in Section IV by a comparison of multihop CTP performance to a star topology protocol under static conditions. Section $\mathrm{V}$ extends the experiments into dynamic scenarios. We conclude with overall thoughts in Section VI.

\section{BACKGROUND}

\section{A. Intra-car wireless sensor networking}

Several intra-car WSN experiments have been conducted for the $915 \mathrm{MHz}$ and $2.4 \mathrm{GHz}$ ISM bands, since many off-the-shelf 


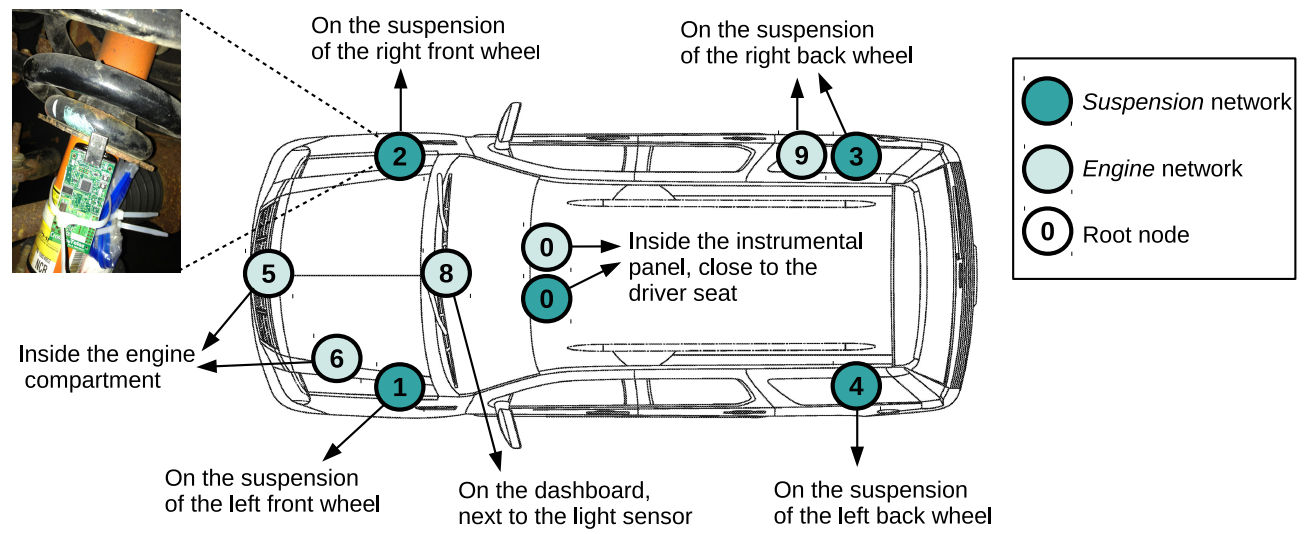

Fig. 1. Placement of nodes inside the car for the suspension network and the engine network.

products (ZigBee, Bluetooth, RFID) operate at these frequencies. The authors in [3] characterize the physical layer of a Zigbee-based sensor network inside a car; in particular, they measure the Received Signal Strength Indicator (RSSI) and Link Quality Indicator (LQI). The results in $[4,9,10]$ provide comprehensive statistics for the intra-car channel, including its power delay profile (PDP), coherence bandwidth and coherence time. Other fading statistics, such as level-crossing rate (LCR) and average fading duration (AFD), have also been measured in these works. In [5], ultra-wideband (UWB) technology is considered for short range communication within a car due to its low power requirements and high data rate.

In contrast to the previous works, we investigate the performance of an intra-car multi-hop WSN on a commercial TelosB platform. Multi-hop data collection can potentially compensate for large channel losses. For example, the results in [10] show that the average channel loss of a link between a transmitter (located in the engine compartment) and a receiver (placed inside the trunk) is about $85 \mathrm{~dB}$. Considering the receiver sensitivity of the sensor's radio chip (e.g., the CC2420 has a sensitivity of $-94 \mathrm{dBm}(\mathrm{typ})$ and $-90 \mathrm{dBm}(\mathrm{min})$ ), one can conclude that sensor nodes should always transmit at high power to overcome such channel loss. On the other hand, the situation is different for a multi-hop network, wherein sensor nodes can opportunistically choose the next hop according to on-going channel conditions and decrease the transmission power while communicating reliably, possibly at the expense of increased latency and decreased network throughput. Our work thus aims to concretely evaluate the cost-benefit regions of multi-hop WSN inside cars.

\section{B. Collection Tree Protocol}

The Collection Tree Protocol (CTP) is a variant of a distancevector routing protocol that includes optimization tailored for wireless sensor networks. CTP is designed to route data from every node on a network to one or more self-declared root nodes, based on minimum cost trees. In [6] a variant of CTP is introduced, in which the expected number of transmissions, denoted by ETX, is used as the cost metric. In this approach, each node that has a message to transmit attempts to build a shortest-path tree with a minimum number of transmissions toward the root. The calculation of ETX for each node is done as follows: consider two nodes A and B such that node A is the parent of node $\mathrm{B}$, then we have:

$$
\begin{gathered}
\mathrm{ETX}_{\mathrm{B}}=\mathrm{ETX}_{\mathrm{A}}+\mathrm{ETX} \text { of link B } \rightarrow \mathrm{A} ; \\
\mathrm{ETX}_{\text {Root }}=0,
\end{gathered}
$$

where the ETX of a link is estimated by a link estimator in a distributed fashion [6]. Given a choice of valid routes, CTP simply chooses the one with the lowest ETX toward the root.

\section{EXPERIMENTAL SETUP}

\section{A. Experimental methodology}

In the experiments, we evaluate two distinct networks operating on different frequencies inside a car: a suspension network and an engine network. Each network consists of four sensor nodes periodically sending data to the collection root, which itself is connected to a laptop through a USB port for the purposes of logging messages and statistics for post-experiment analyses. Fig. 1 graphically illustrates the placement of nodes in the car. Note that previous studies (see, for example, [10]) show that wireless channels to other locations in the vehicle show more-or-less similar characteristics, i.e., slow fading with coherence time of several seconds.

In addition to the sensor nodes and the collection root, we utilize an activator node (not shown in Fig. 1) to send an initial broadcast signal that activates each sensor node and establishes basic time synchronization. The use of an activation broadcast enables us to dictate the packet generation rate, transmission power, and radio channel for the nodes. The activator node is chosen to be different from the root node because all nodes should receive the activation signal, while the signal of the root may not be received by all nodes under low-power transmission. Therefore, we use the activator node to send a high-power broadcast signal received by all sensors.

Our sensor nodes are TelosB (TPR2420CA) [11] with a data rate of $250 \mathrm{kbps}$, RF power $-24 \mathrm{dBm}$ to $0 \mathrm{dBm}$, and 
TABLE I

EXPERIMENTAL SCENARIOS

\begin{tabular}{|c|c|c|c|c|}
\hline \multirow{2}{*}{ Location } & \multirow{2}{*}{ Scenario } & \multicolumn{3}{|c|}{ Dynamic conditions } \\
\hline & & Engine & Passengers & $\mathrm{WiFi}$ \\
\hline \multicolumn{5}{|l|}{ Parking } \\
\hline \multirow{4}{*}{ Covered area } & Static & Off & No & Weak $^{1}$ \\
\hline & Engine-on & On & No & Weak \\
\hline & Passengers & Off & Yes & Weak \\
\hline & WiFi interference & Off & No & Strong $^{2}$ \\
\hline Open area & Static & Off & No & Weak \\
\hline \multicolumn{5}{|l|}{ Driving } \\
\hline \multirow{3}{*}{ On the road } & Bumpy road & On & Yes & Weak \\
\hline & Highway & On & Yes & Weak \\
\hline & Urban area & On & Yes & Strong $^{3}$ \\
\hline
\end{tabular}

\footnotetext{
${ }^{1}$ In-band WiFi interference is negligible.

${ }^{2}$ Controlled WiFi interference is exerted to the experiment setup.
}

${ }^{3}$ There exist considerable urban wireless interferences.

receiver sensitivity of $-94 \mathrm{dBm}$ (typ) through $-90 \mathrm{dBm}$ (min). The sensor nodes' firmware is based on TinyOS 2.x, which is an open source operating system designed for sensor networks. The radio chip of sensor nodes (CC2420) is configured to use a Carrier Sense Multiple Access (CSMA) MAC protocol for transmission through the shared wireless medium.

We investigate CTP performance under several conditions, summarized in Table I. The experiments are performed in (i) a covered area parking with little foot traffic and several cars parked nearby, (ii) an open area parking, and (iii) a local road, highway, or an urban area. The condition of an experiment can be either static or dynamic, with the latter referring to cases when the engine is on, or passengers move in and out of the vehicle, or WiFi interferences are present.

\section{B. Sensors data collection}

In real deployment of intra-car WSNs, data packets contain physical measurements of sensors such as tire pressure in a Tire Pressure Monitoring System (TPMS), engine torque, or transmission pressure. For instance, a Transmission Control Module (TCM) controls automatic transmissions in modern vehicles. TCM uses readings from transmission sensors as well as data provided by the ECUs to change gears for optimum performance in terms of fuel efficiency and shift quality [12]. In the experiments, however, we have abstracted the payload of packets (shown in Fig. 2) into information that is needed to calculate performance metrics of the network. For instance, when a sensor node receives a packet, it updates payload of the packet with some metadata (e.g., hop ID and RSSI information) and forwards it to the next hop. Payload fields are then processed in a post-experiment step using our developed toolkit in [13] to calculate the performance metrics and display the evolution of the network topology. In the experiments, sensor nodes generate packets with a size of 32 bytes including 8 bytes of CTP data packet header, 12 bytes of payload, and 12 bytes of CC2420 header.

\begin{tabular}{|l|l|l|l|l|l|l|l|l|l|l|l|l|l|l|l|}
\hline 0 & 1 & 2 & 3 & 4 & 5 & 6 & 7 & 8 & 9 & 10 & 11 & 12 & 13 & 14 & 15 \\
\hline \multicolumn{3}{|c|}{ src_node_id } & \multicolumn{3}{|c|}{ hop_count } \\
\hline 1st hop_node_id & \multicolumn{3}{|c|}{ 1st hop_rssi } \\
\hline 2nd hop_node_id & \multicolumn{3}{|c|}{ 2nd hop_rssi } \\
\hline 3rd hop_node_id & \multicolumn{3}{|c|}{ 3rd hop_rssi } \\
\hline 4th hop_node_id & \multicolumn{3}{|c|}{ 4th hop_rssi } \\
\hline \multicolumn{4}{|c|}{ packet_id } \\
\hline \multicolumn{4}{|c|}{}
\end{tabular}

Fig. 2. Payload fields of a packet

Throughout the experiments, we measure the average delivery rate, delay, and number of transmissions per packet, denoted by Tx count. Assuming that in an experiment lasting $T$ seconds, sensors generate $M$ packets in total from which $N$ packets are successfully received by the root. Among the total $N$ received packets, $N_{u}$ packets are unique (i.e., all duplicates are removed from the count). The delivery rate is then defined as the ratio of uniquely received packets to the total number of generated packets $\left(\frac{N_{u}}{M} \times 100 \%\right)$. The delay of a delivered packet is the time elapsing from its generation at the source node till its reception at the root. Tx count is the number of total transmissions (including at relay nodes) per received packet at the root.

\section{Experimental Results: Static CONDitions}

In this section, we compare the performance of CTP with a single-hop star protocol under static conditions.

\section{A. Reliability}

In a star protocol all nodes communicate directly with the root, and thus, if some node-to-root links experience deep channel fading then the quality of service for that node degrades, while an intra-car WSN should guarantee high reliability across all nodes. For instance, in a TPMS network, each sensor node attached to a tire should be able to successfully deliver measured parameters to the central processors. Within this context, we examine the delivery rate of individual nodes using CTP and the star protocol. As the results in Fig. 3 show, CTP provides high delivery rate across all nodes, whereas the performance of the star protocol varies significantly. For example, node 4 has a delivery rate of $78 \%$ with high variance showing that nodes can have varying performance over time depending on the link conditions. From the network topology shown in Fig. 4, we observe that the topology induced by CTP is indeed multi-hop, in counterpoint to the single-hop topology of the star protocol.

\section{B. Communication and latency trade-offs}

In wireless sensor networks power consumption and delay have significance for battery-powered and delay-limited applications. While comparing multi-hop with single-hop, some trade-offs emerge: a multi-hop topology may require more transmissions per packet due to relay nodes, but nodes can transmit with lower power to achieve the same delivery rate as a single-hop protocol. For instance, our experimental results show that CTP with a transmit power of $-10 \mathrm{dBm}$ can provide 


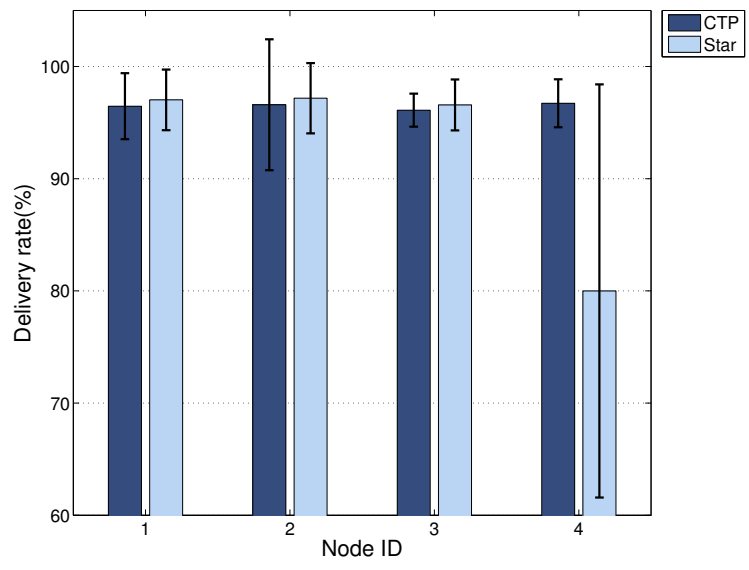

Fig. 3. Delivery rate of CTP vs. the star protocol under static conditions, Tx power $-20 \mathrm{dBm}$ and generation rate $15 \mathrm{pkts} / \mathrm{sec}$ per node. The error bars show one standard deviation from the mean over 5 six-minutes experiments.

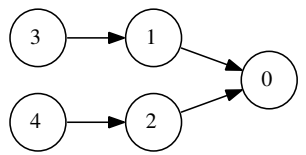

(a) CTP topology

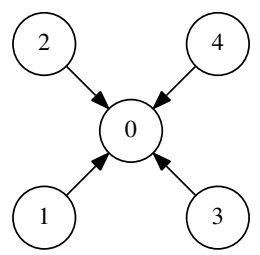

(b) Star topology
Fig. 4. CTP multi-hop topology vs. the single-hop topology of the star protocol

the same reliability as the star protocol with a transmit power of $0 \mathrm{dBm}$, at the cost of only up to $69 \%$ higher Tx count per packet. Specifically, the radio of TelosB motes (CC2420) draws $11 \mathrm{~mA}$ current to transmit at a power of $-10 \mathrm{dBm}$ and 17.4 $\mathrm{mA}$ to transmit at a power of $0 \mathrm{dBm}$ [14]. On the other hand, the receive mode requires a current of $18.8 \mathrm{~mA}$, noting that in our application, the radio is almost always listening for incoming messages, which implies that the radio consumes roughly the same amount of receive power regardless of transmission activity. Based on the number of transmissions and transmit power, we conclude that CTP provides energy savings for the radio component. Previous experiments and simulations in [15] show that energy consumption by the radio is dominant in wireless sensor motes. However, it should be noted that other components, such as the processor, consume energy, and that their energy consumption depends on the instructions run by the mote.

Single-hop delay is also expected to be smaller than multihop delay, but a single-hop protocol is not always reliable and retransmissions are required. Table II compares the average Tx count and delay performance of CTP and the star protocol. The results confirm that a star protocol requires fewer transmissions and incurs lower delay, but CTP provides a higher delivery rate. While the average delivery rate of the star protocol may not be significantly worse than CTP, individual nodes can experience a low delivery rate within the star protocol (e.g., node 4 in Fig. 3).

\begin{tabular}{cccccc}
\hline Condition & Tx power & Protocol & Tx count & Delay & Delivery \\
\hline \multirow{2}{*}{ Static } & \multirow{2}{*}{$-20 \mathrm{dBm}$} & Star & 1.14 & 14.36 & $92.69 \%$ \\
& & CTP & 1.72 & 37.35 & $96.43 \%$ \\
\hline \multirow{2}{*}{ Passengers } & $-10 \mathrm{dBm}$ & Star & 1.22 & 16.72 & $92.59 \%$ \\
& & CTP & 2.54 & 82.92 & $98.41 \%$ \\
\hline
\end{tabular}

TABLE II

CTP AND THE STAR PROTOCOL PERFORMANCE. TX COUNT AND DELAY ARE CALCULATED BASED ON THE DELIVERED PACKETS.

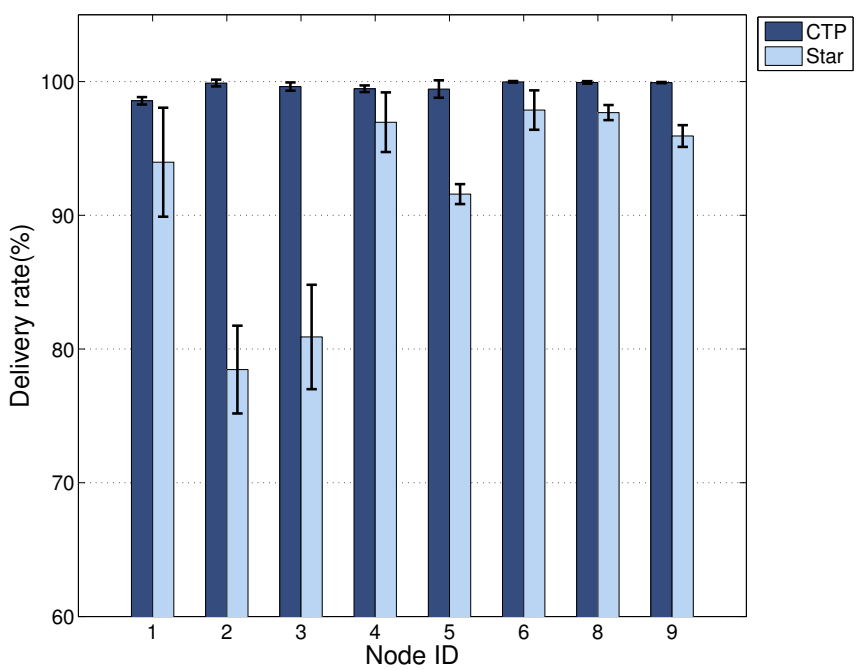

Fig. 5. Delivery rate of CTP vs. the star protocol under static conditions for the 8-nodes network. The packet generation rate is $5 \mathrm{pkts} / \mathrm{sec}$ per node and the transmission power is set to $-10 \mathrm{dBm}$.

\section{Reliability in larger network}

To generalize the previous reliability results, we merge the suspension network and the engine network into one network with eight sensors and one collection root (see Fig. 1). From the delivery rate results shown in Fig. 5, one can notice that the performance of CTP is more reliable and stable across all nodes. In fact, different nodes in the star protocol achieve varying levels of reliability, but CTP reduces variance in performance among the different nodes and all of them achieve a delivery rate higher than $98 \%$.

\section{EXPERIMENTAl Results: DynAmic CONDitions}

We next investigate the performance of CTP and the star protocol under dynamic in-vehicle conditions.

\section{A. CTP vs. Star protocol under dynamic conditions}

An intra-car wireless sensor network can experience various environmental conditions, such as when the car travels through areas with intense wireless interference, or when passengers are sitting inside the vehicle, which can cause link fluctuations. However, an intra-car WSN should provide robust performance regardless of environmental conditions. Within this context, we compare the performance of CTP and the star protocol 


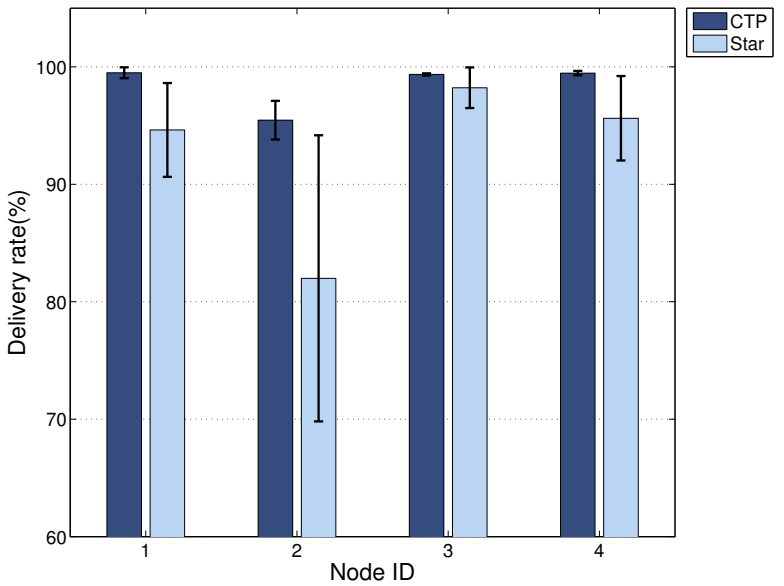

(a) Passengers-move-in-and-out scenario

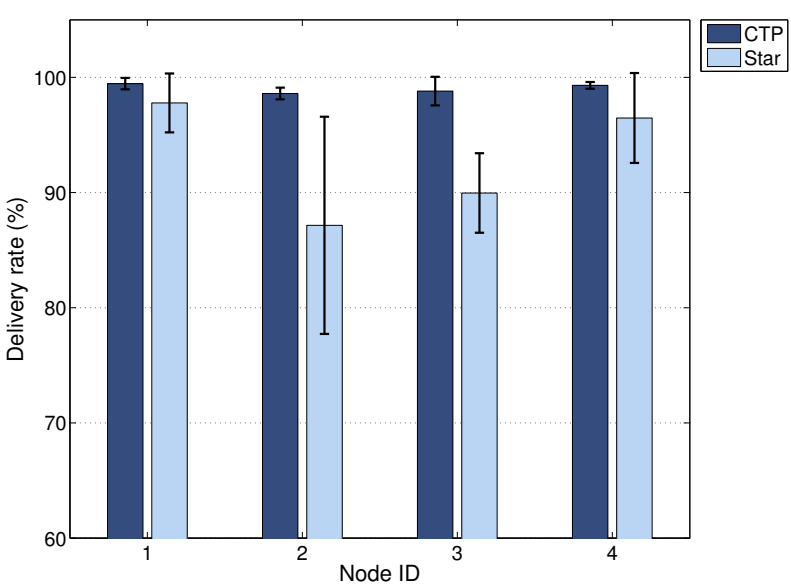

(b) WiFi interference

Fig. 6. Delivery rate of CTP vs. the star protocol (a) under Passengers-move-in-and-out scenario (b) with WiFi interferences, Tx power - 10 dBm and generation rate $10 \mathrm{pkts} / \mathrm{sec}$ per node. The error bars show one standard deviation from the mean over 5 six-minutes experiments.

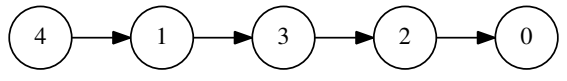

(a) Passengers-move-in-and-out scenario

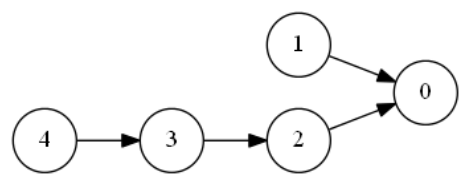

(b) WiFi interference

Fig. 7. Multi-hop CTP topology under (a) passengers-move-in-and-out scenario (b) with WiFi interference.

under two common dynamic scenarios: (i) when passengers are sitting inside the vehicle, and (ii) in the presence of Wi-Fi interferences. Fig. 17(a) shows the delivery rate of individual nodes when two passengers move in and out of the vehicle. The results indicate that CTP provides stable and reliable performance across all nodes, whereas the star protocol has worse delivery rate. For instance, only $82 \%$ of the packets generated at node 2 are successfully received by the root. Fig. 17(b) compares the performance of CTP and the star protocol in the presence of WiFi interferences. Likewise, we observe that there are individual nodes within the star protocol that fail to achieve high delivery rate. The network topology induced by CTP under dynamic conditions is shown in Fig. 7, which confirms that multi-hop topology can provide robust performance under dynamic conditions.

\section{B. CTP supplementary experiments}

In this part, we extend the experiments of CTP to investigate its performance sensitivity to various environmental conditions.

Open area vs. covered area: Due to the broadcast nature of wireless signals, the presence of surrounding objects, like walls and ceilings, affects wireless network performance. Within this
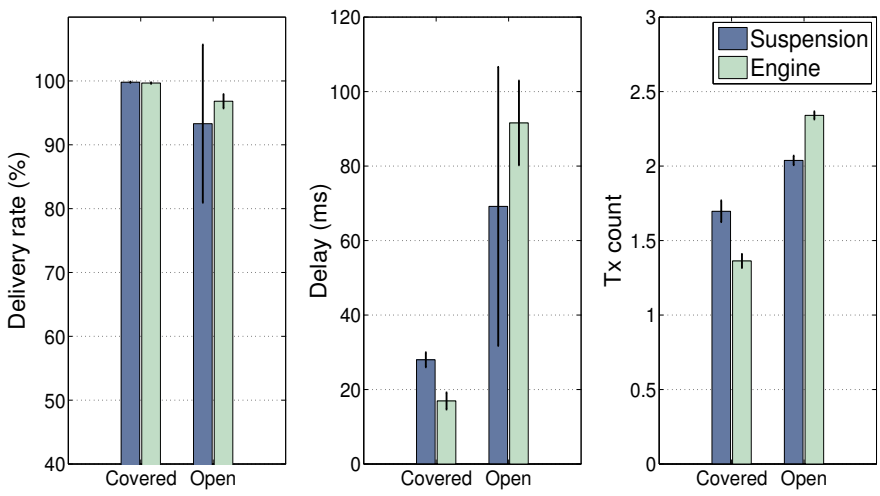

Fig. 8. CTP performance of the suspension network and the engine network in a covered and an open area parking with Tx power $-10 \mathrm{dBm}$ and the packet generation rate $10 \mathrm{pkts} / \mathrm{sec}$ per node.

context, CTP performance is examined in both covered and open area parking. Results shown in Fig. 8 indicate that CTP achieves better performance in the covered area parking. We speculate that this could be due to more multi-path signaling in the covered area, which adds up to create a strong almostconstant signal at the receiver; an open area is potentially a poor multi-path environment, which is consistent with the experimental results in [10].

Engine-on, with Passengers: Physical channels inside a car can be highly dynamic due to external disturbances such as when the car engine is on, or when passengers move in and out of the vehicle. Fig. 9 shows the performance of the suspension network under such conditions. The results illustrate that CTP achieves a delivery rate above $98 \%$, and that engine noise does not have considerable effect on overall system performance. However, delay and Tx count performance degrade with passengers movements, indicating that human body can cause channel fading that may be attributed to the large attenuation of biological tissues in the $2.4 \mathrm{GHz}$ range [16].

Driving experiments: Data collection from intra-car wire- 

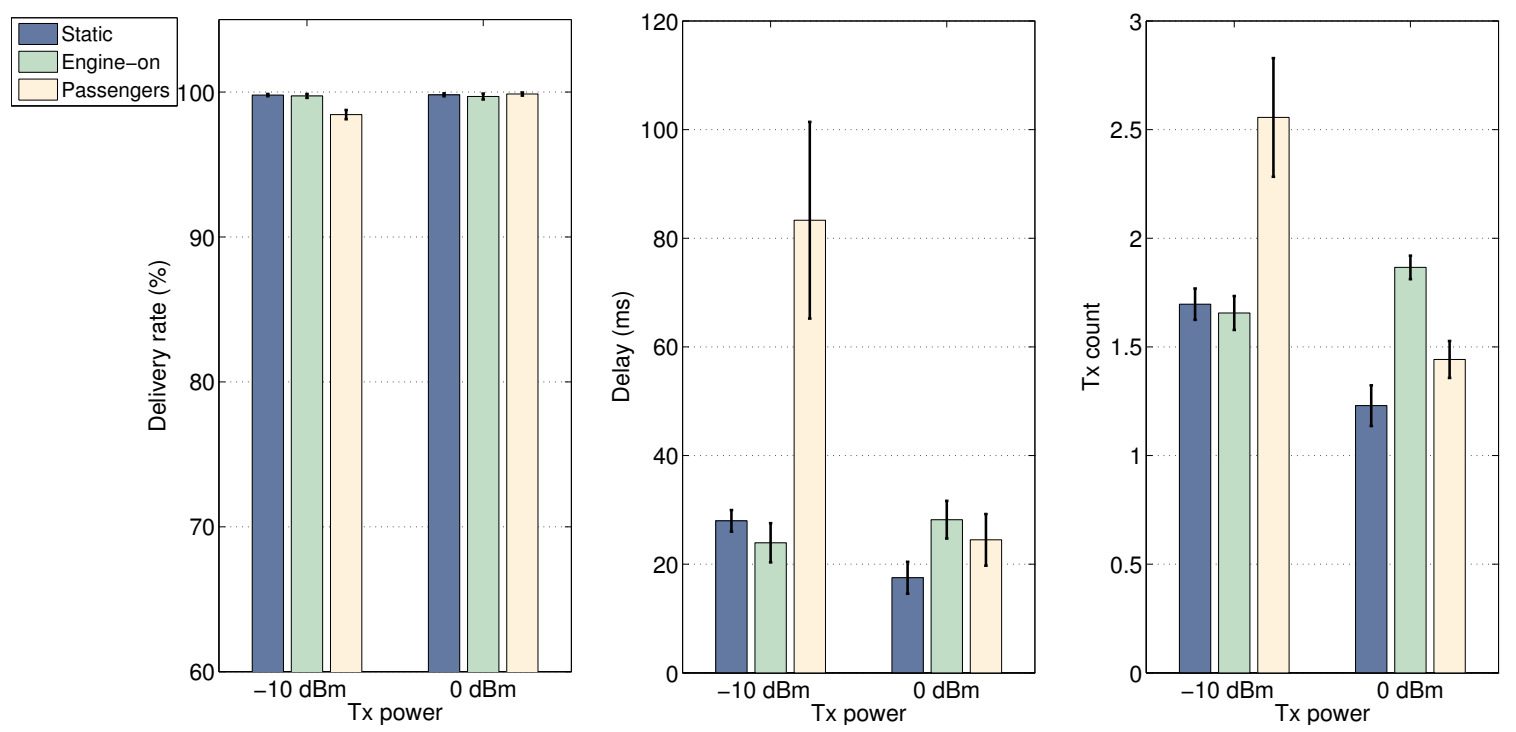

Fig. 9. CTP performance under engine-on and passengers-move-in-and-out conditions for the suspension network and the packet generation rate 10 pkts/sec per node.
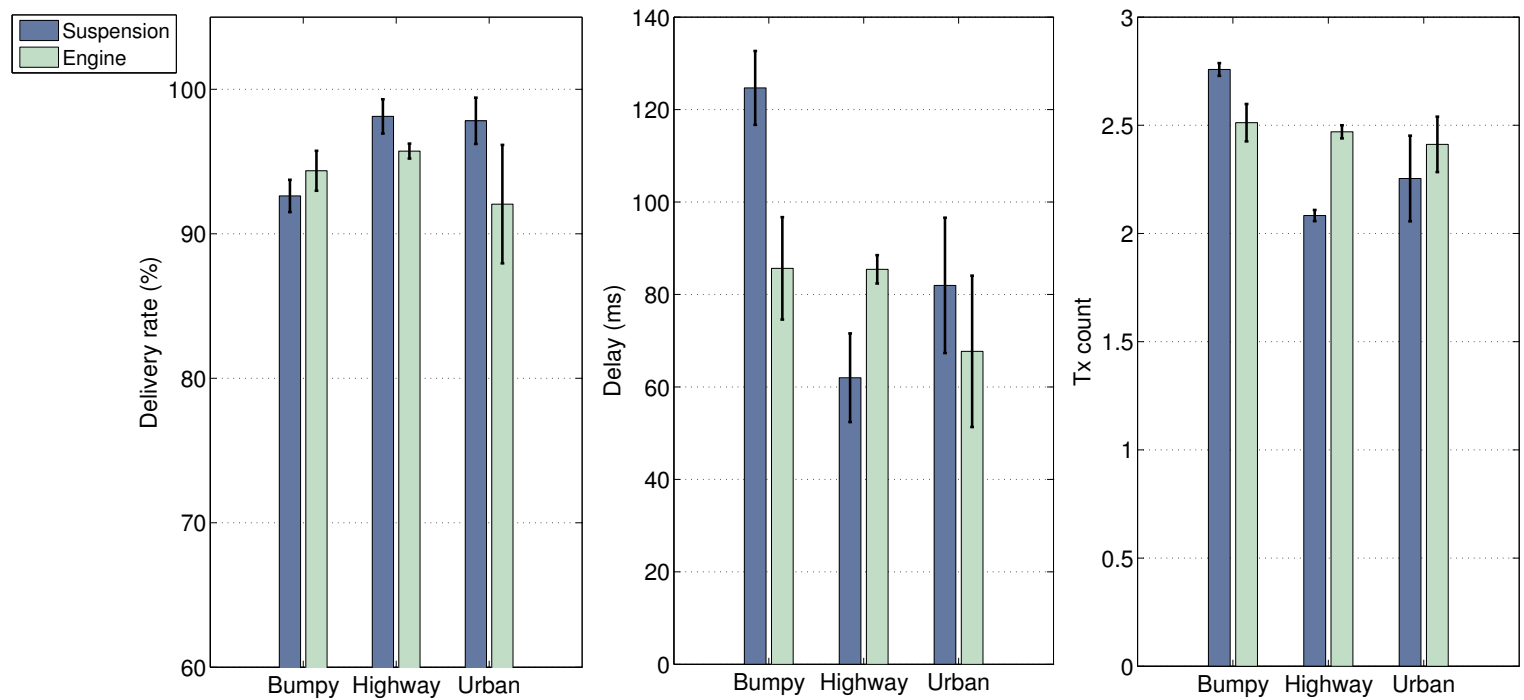

Fig. 10. CTP performance of the suspension network and the engine network within various driving conditions. Tx power is set to $-10 \mathrm{dBm}$ and the packet generation rate is $10 \mathrm{pkts} / \mathrm{sec}$ per node.

less sensors is complicated by the variety of conditions a car experiences. As such, driving experiments play a critical role in performance assessment of an intra-car WSN. To fulfill this evaluation goal, CTP experiments are performed within three driving conditions: (i) bumpy road with poor road quality, (ii) highway with sparse and high speed vehicular traffic, and (iii) urban area with dense vehicular traffic and wireless interference (WiFi, Bluetooth, etc.). Driving results shown in Fig. 10 illustrate that both networks have a delivery rate higher than $90 \%$ under various driving conditions. The performance of the suspension network, whose sensor nodes are attached to the suspension system of wheels, is most affected when driving on the bumpy road. High speed driving on a highway does not have noticeable effect on both networks and driving in urban areas does not have considerable effect on the suspension network either, but the delivery rate of the engine network degrades in urban areas, presumably due to wireless interferences.

WiFi interference: Sensor radios based on the IEEE 802.15.4 standard can highly suffer from external interferences, as they use the same frequency band as the IEEE 802.11 standard. To investigate the performance of CTP under interferences, we run a series of controlled WiFi experiments, wherein sensor nodes are configured to operate on the same frequency as a local WiFi network (channel 22 under 802.15.4 standard and channel 11 of 802.11 standard). External interferences are applied within two intensity levels: (i) light WiFi that reflects a "normal" WiFi usage by local users, and (ii) heavy WiFi that is exerted by streaming a video on a close 

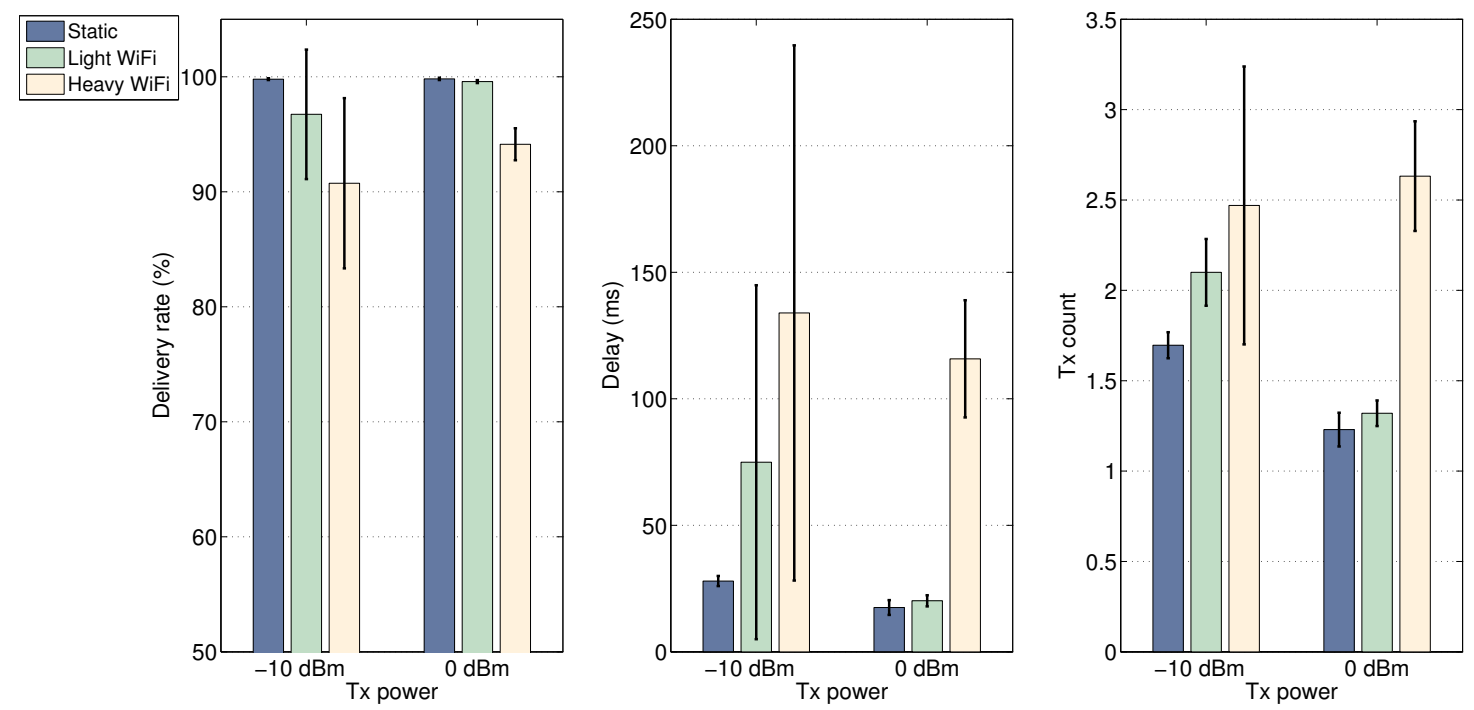

(a) Performance of the suspension network under WiFi interference
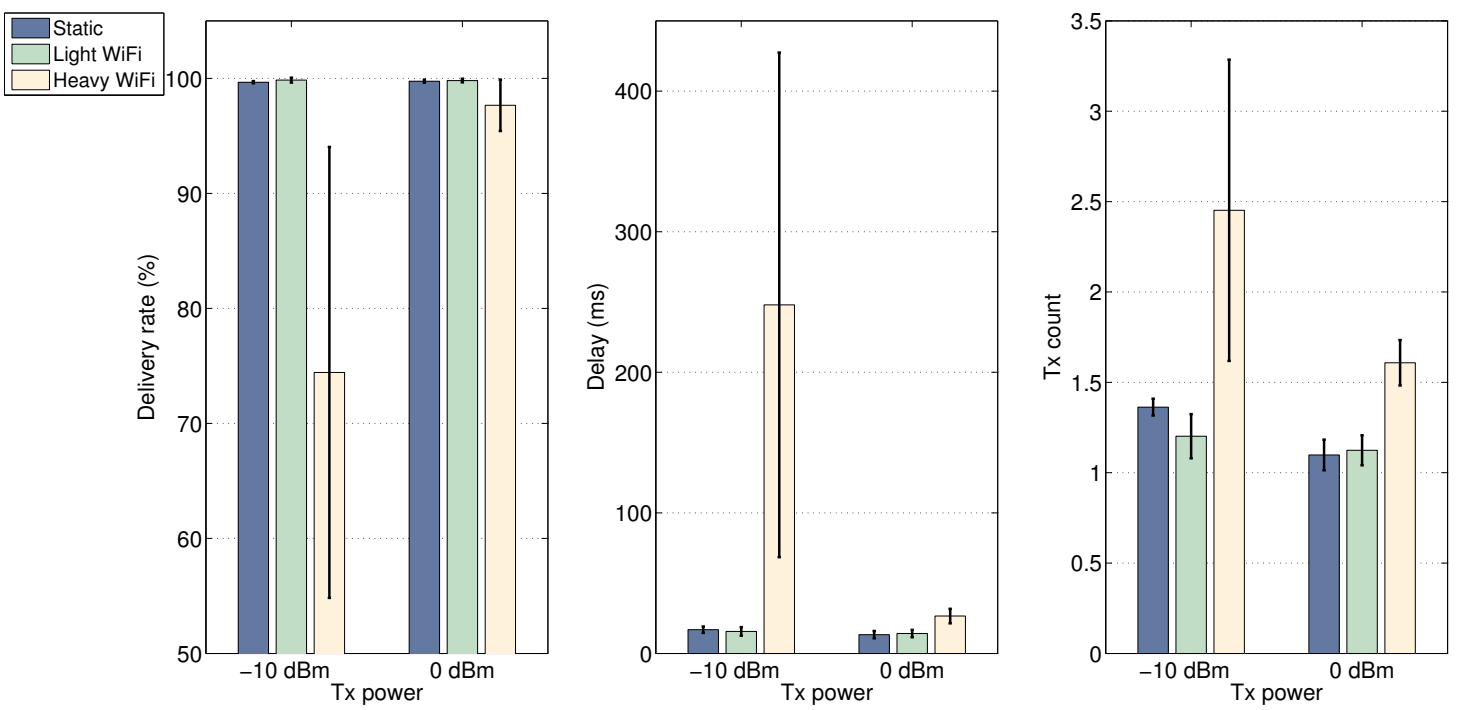

(b) Performance of the engine network under WiFi interference

Fig. 11. CTP performance under external WiFi interference for (a) the suspension network and (b) the engine network with the packet generation rate 10 pkts/sec per node.

proximity computer located on the passenger's seat. From the results shown in Fig. 18(b), we observe that the engine network is more vulnerable to WiFi interference, especially with low transmission power. This observation is consistent with driving experiment results in the urban area with wireless interferences. It is also evident that intense WiFi interference deteriorates both networks' performance due to low SNR at the nodes' receivers.

\section{CONCLUSION}

In this article we have investigated the performance of CTP as a multi-hop data collection approach, as a counterpoint to the star protocol that dominates the existing literature for intra-car wireless sensor networks. Through experiments, we demonstrate that the delivery rate of the star protocol can be low in practical scenarios, while CTP achieves a reliability of more than $90 \%$ across all nodes. Whereas a star protocol requires fewer transmissions and incurs lower average delay than CTP, the radio energy consumption of CTP is smaller. Our experimental results indicate that environmental conditions have widely differing effects on network performance. For instance, passengers cause channel fading and degrade overall system performance, while engine noise on the order of 2$4 \mathrm{~dB}$ does not have noticeable effect on performance. External intense interferences (WiFi, Bluetooth, etc.) potentially deteriorate network performance, but CTP sustains its high delivery rate (higher than 90\%) in various driving conditions. These results serve as an illustration of the cost-benefit regions of multi-hop WSN inside cars.

Overall, our experimental results show that multi-hop networking enhances many aspects of network performance, and may be suitable for intra-car networks due to their need for 
robust operation in harsh environments. In future works, we plan to explore hybridized collection protocols that blend reliability with other desired performance metrics, like throughput optimality.

\section{ACKNOWLEDGEMENT}

The work presented here was supported by General Motors, Advanced Technical Center, Israel.

\section{BIOGRAPHIES}

Morteza Hashemi (mhashemi@bu.edu) received his B.Sc. (2011) in Electrical Engineering from Sharif University of Technology, Tehran, Iran. He is currently a PhD student in Electrical and Computer Engineering at Boston University. His research interests include error correcting code, networks performance evaluation, and wireless communications.

Wei Si (weisi@bu.edu) received B.S. degree from Shanghai Jiao Tong University, Shanghai, China, in 2010. Currently, he is working towards his Ph.D. degree in Systems Engineering at Boston University. His research interests include routing protocols for intra-car wireless sensor networks and disruption tolerant networks, data synchronization algorithms and queueing theory.

Moshe Laifenfeld (moshe.laifenfeld@gm.com) received his BSc from the Technion in 1992, his MSc from Tel-Aviv University in 1998, and his Ph.D. from Boston University in 2008, all in electrical and computer engineering. Moshe then took a joint post-doctoral position at MIT and Boston University, where he focused on aspects of coding theory in communication networks. Since then he is with General Motors, focusing on $\mathrm{R} \& \mathrm{D}$ of novel hybrid in-vehicle electrical architectures. In his past, Moshe led the algorithms development of a 3rd generation UMTS transceiver, and held several $R \& D$ positions in medical devices start-ups.

David Starobinski (staro@bu.edu) is a Professor of Electrical and Computer Engineering at Boston University, with a joint appointment in the Division of Systems Engineering. He received his Ph.D. in Electrical Engineering from the Technion - Israel Institute of Technology, in 1999. His research interests are in wireless networking, network economics, and cybersecurity.

Ari Trachtenberg (trachten@bu.edu) is a Professor of Electrical and Computer Engineering at Boston University. He received his Ph.D. (2000) and M.S. (1996) in Computer Science from the University of Illinois at Urbana/Champaign, and his S.B. from MIT (1994) in math with CS. His research interests include cybersecurity (smartphones, cryptography), networking (security, sensors, localization), algorithms (data synchronization, file edits, file sharing), and error-correcting codes (rateless coding, feedback).

\section{REFERENCES}

[1] CHOSeN: Cooperative hybrid objects sensor networks http://www.chosen.eu.

[2] Mathias Grudén, Alexander Westman, Janis Platbardis, P Hallbjorner, and Anders Rydberg. Reliability experiments for wireless sensor networks in train environment. In Wireless Technology Conference, EuWIT, pages 37 40. IEEE, 2009.

[3] Hsin-Mu Tsai, Ozan K Tonguz, Cem Saraydar, Timothy Talty, Michael Ames, and Andrew Macdonald. Zigbee-based intra-car wireless sensor networks: a case study. Wireless Communications, IEEE, 14(6):67-77, 2007.

[4] Ozan K Tonguz, Hsin-Mu Tsai, Timothy Talty, Andrew Macdonald, and Cem Saraydar. RFID technology for intra-car communications: a new paradigm. In Vehicular Technology Conference, VTC Fall. IEEE 64th, pages 1-6, 2006.

[5] W. Niu, J. Li, and T. Talty. Intra-vehicle uwb channels in moving and staionary scenarios. In Military Communications Conference, 2009. MILCOM 2009. IEEE, pages 1-6. IEEE, 2009.

[6] Omprakash Gnawali, Rodrigo Fonseca, Kyle Jamieson, Maria Kazandjieva, David Moss, and Philip Levis. CTP: An efficient, robust, and reliable collection tree protocol for wireless sensor networks. $A C M$ Transactions on Sensor Networks (TOSN), 10(1):16, 2013.

[7] Tim Winter. RPL: IPv6 routing protocol for low-power and lossy networks. 2012.

[8] Morteza Hashemi, Wei Si, Moshe Laifenfeld, David Starobinski, and Ari Trachtenberg. Intra-car wireless sensors data collection: A multi-hop approach. In Vehicular Technology Conference, VTC Spring. IEEE 77th, pages 1-5, 2013.

[9] Hsin-Mu Tsai, Wantanee Viriyasitavat, Ozan K Tonguz, Cem Saraydar, Timothy Talty, and Andrew Macdonald. Feasibility of in-car wireless sensor networks: A statistical evaluation. In Sensor, Mesh and Ad Hoc Communications and Networks. SECON'07. 4th Annual IEEE Communications Society Conference on, pages 101-111, 2007.

[10] Amir R Moghimi, Hsin-Mu Tsai, Cem U Saraydar, and Ozan K Tonguz. Characterizing intra-car wireless channels. Vehicular Technology, IEEE Transactions on, 58(9):5299-5305, 2009.

[11] MEMSIC. Telosb specification. http://www.memsic.com.

[12] Kobi Vaknin, Moshe Laifenfeld, Leor Hardy, and Aviv Goll. Experimenting with a wireless mesh network towards sensing inside a vehicle's transmission. In IEEE International Conference on Microwaves, Communications, Antennas and Electronics Systems (COMCAS), pages 1-5, 2013.

[13] Wei Si, Morteza Hashemi, Idan Warsawski, Moshe Laifenfeld, David Starobinski, and Ari Trachtenberg. TeaCP: A toolkit for evaluation and analysis of collection protocols in wireless sensor networks. In IEEE International Conference on Microwaves, Communications, Antennas and Electronics Systems (COMCAS), pages 1-5, 2013.

[14] CC2420 radio. http://www.ti.com.cn/cn/lit/ds/symlink/cc2420.pdf.

[15] Aggeliki S. Prayati, Christos Antonopoulos, Tsenka Stoyanova, Christos Koulamas, and George D. Papadopoulos. A modeling approach on the TelosB WSN platform power consumption. Journal of Systems and Software, 83(8):1355-1363, 2010.

[16] Julien Ryckaert, Philippe De Doncker, René Meys, Arnaud de Le Hoye, and Sophie Donnay. Channel model for wireless communication around human body. Electronics letters, 40(9):543-544, 2004. 


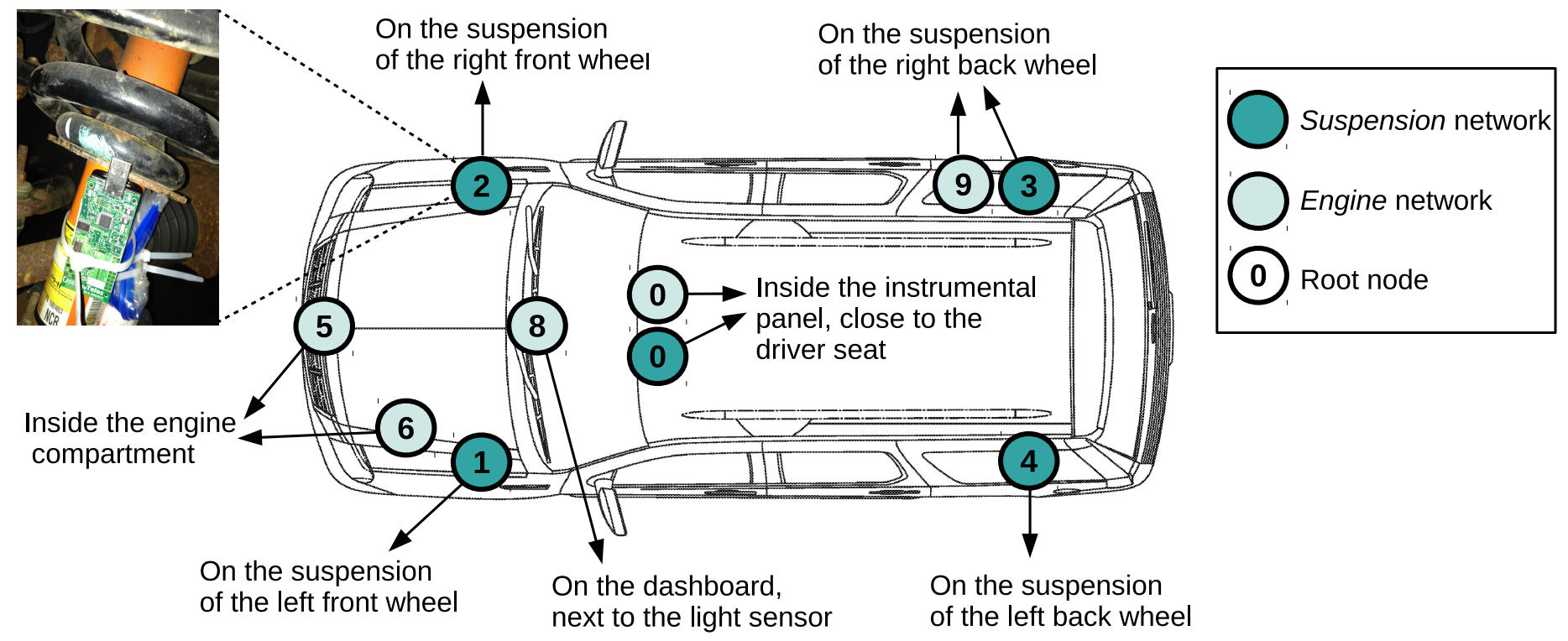

Fig. 12. Placement of nodes inside the car for the suspension network and the engine network. 
TABLE III

EXPERIMENTAL SCENARIOS

\begin{tabular}{|c|c|c|c|c|}
\hline \multirow{2}{*}{ Location } & \multirow{2}{*}{ Scenario } & \multicolumn{3}{|c|}{ Dynamic conditions } \\
\hline & & Engine & Passengers & WiFi \\
\hline \multicolumn{5}{|l|}{ Parking } \\
\hline \multirow{4}{*}{ Covered area } & Static & Off & No & Weak $^{1}$ \\
\hline & Engine-on & On & No & Weak \\
\hline & Passengers & Off & Yes & Weak \\
\hline & WiFi interference & Off & No & Strong 2 \\
\hline Open area & Static & Off & No & Weak \\
\hline \multicolumn{5}{|l|}{ Driving } \\
\hline \multirow{3}{*}{ On the road } & Bumpy road & On & Yes & Weak \\
\hline & Highway & On & Yes & Weak \\
\hline & Urban area & On & Yes & Strong \\
\hline
\end{tabular}

\footnotetext{
${ }^{1}$ In-band WiFi interference is negligible.

${ }^{2}$ Controlled WiFi interference is exerted to the experiment setup.

${ }^{3}$ There exist considerable urban wireless interferences.
} 


\begin{tabular}{|l|l|l|l|l|l|l|l|l|l|l|l|l|l|l|l|}
\hline 0 & 1 & 2 & 3 & 4 & 5 & 6 & 7 & 8 & 9 & 10 & 11 & 12 & 13 & 14 & 15 \\
\hline \multicolumn{3}{|c|}{ src_node_id } & \multicolumn{3}{|c|}{ hop_count } \\
\hline \multicolumn{3}{|c|}{ 1st hop_node_id } & \multicolumn{3}{|c|}{ 1st hop_rssi } \\
\hline 2nd hop_node_id & \multicolumn{3}{|c|}{ 2nd hop_rssi } \\
\hline 3rd hop_node_id & \multicolumn{3}{|c|}{ 3rd hop_rssi } \\
\hline 4th hop_node_id & \multicolumn{3}{|c|}{ 4th hop_rssi } \\
\hline \multicolumn{4}{|c|}{ packet_id } \\
\hline \multicolumn{4}{|c|}{}
\end{tabular}

Fig. 13. Payload fields of a packet 


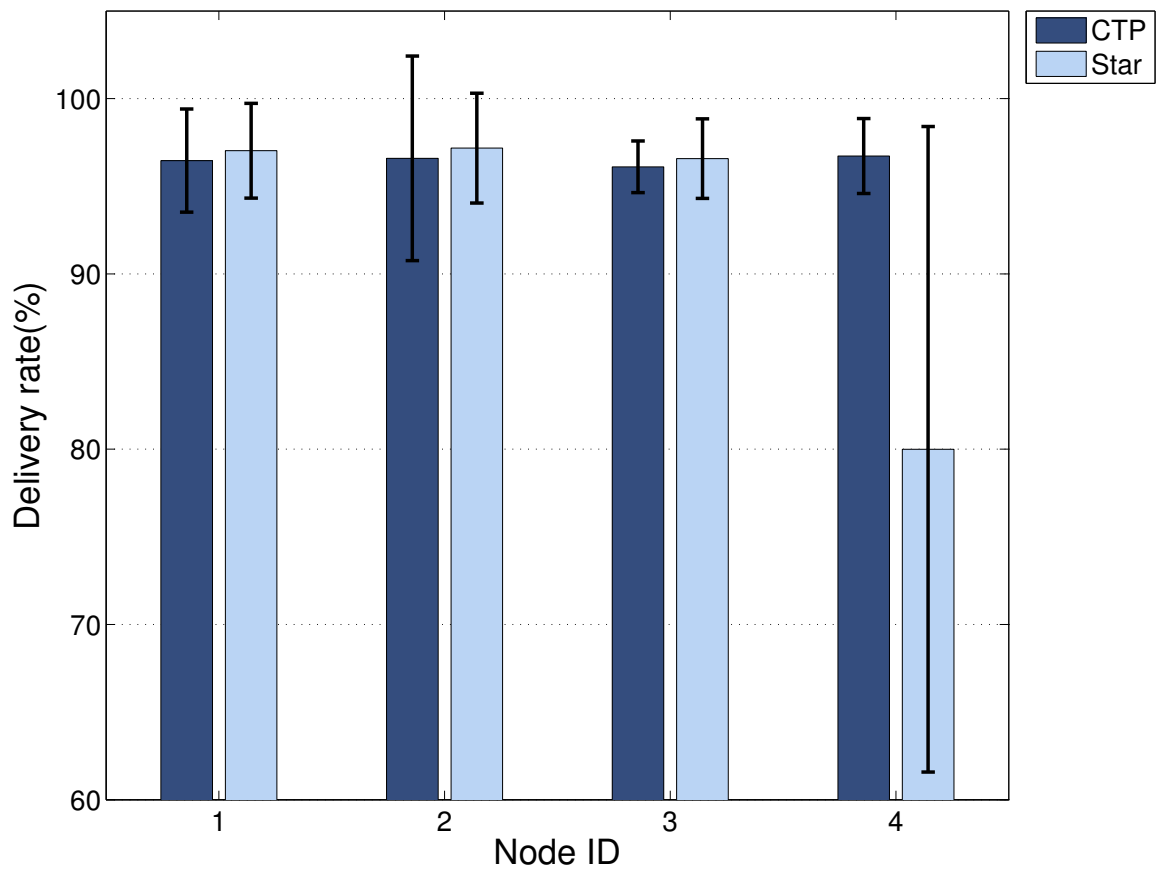

Fig. 14. Delivery rate of CTP vs. the star protocol under static conditions, Tx power $-20 \mathrm{dBm}$ and generation rate 15 pkts/sec per node. The error bars show one standard deviation from the mean over 5 six-minutes experiments. 


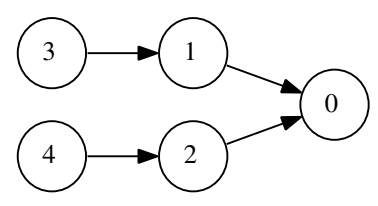

(a) CTP topology

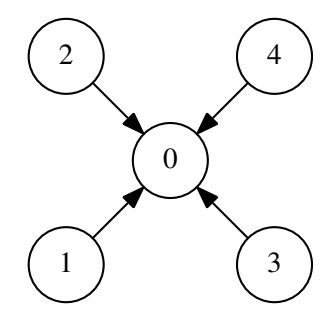

(b) Star topology

Fig. 15. CTP multi-hop topology vs. the single-hop topology of the star protocol 


\begin{tabular}{cccccc}
\hline Condition & Tx power & Protocol & Tx count & Delay & Delivery \\
\hline \multirow{2}{*}{ Static } & \multirow{2}{*}{$-20 \mathrm{dBm}$} & Star & 1.14 & 14.36 & $92.69 \%$ \\
& & CTP & 1.72 & 37.35 & $96.43 \%$ \\
\hline \multirow{2}{*}{ Passengers } & \multirow{2}{*}{$-10 \mathrm{dBm}$} & Star & 1.22 & 16.72 & $92.59 \%$ \\
& & CTP & 2.54 & 82.92 & $98.41 \%$ \\
\hline
\end{tabular}

TABLE IV

CTP AND THE STAR PROTOCOL PERFORMANCE. TX COUNT AND DELAY ARE CALCULATED BASED ON THE DELIVERED PACKETS. 


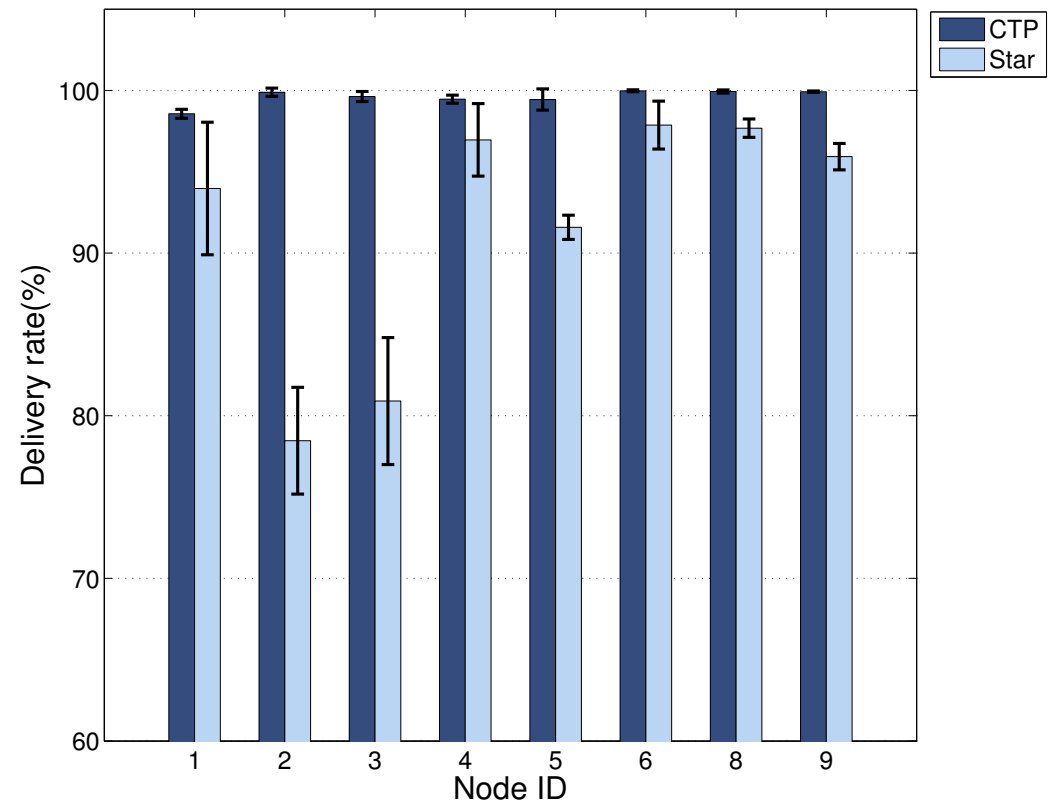

Fig. 16. Delivery rate of CTP vs. the star protocol under static conditions for the 8-nodes network. The packet generation rate is 5 pkts/sec per node and the transmission power is set to $-10 \mathrm{dBm}$. 


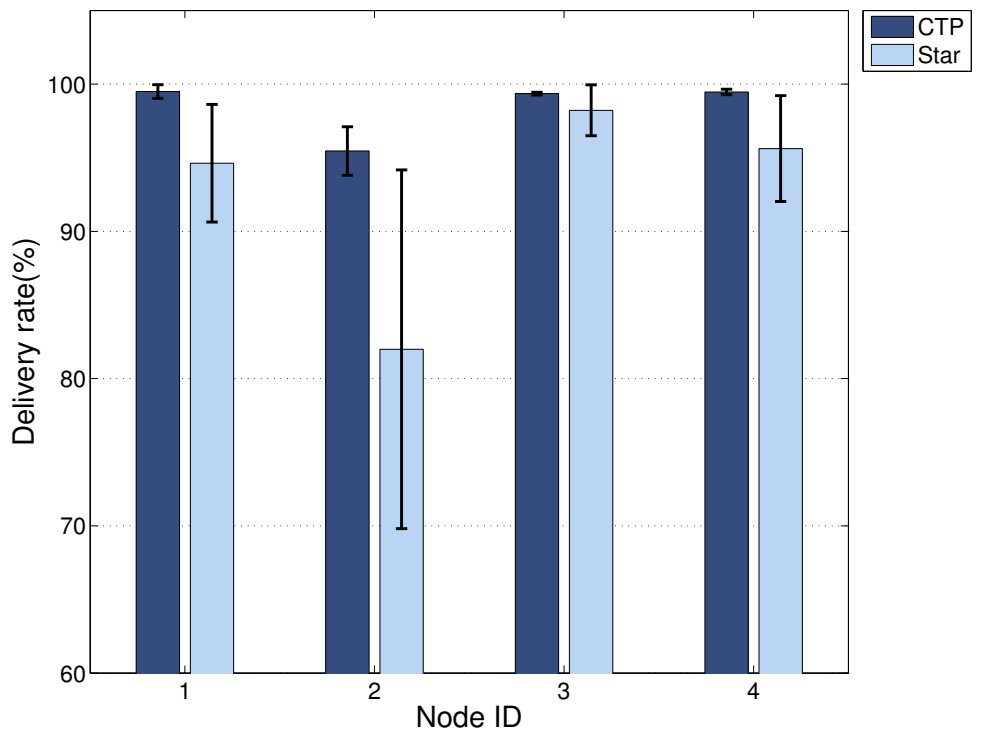

(a) Passengers-move-in-and-out scenario

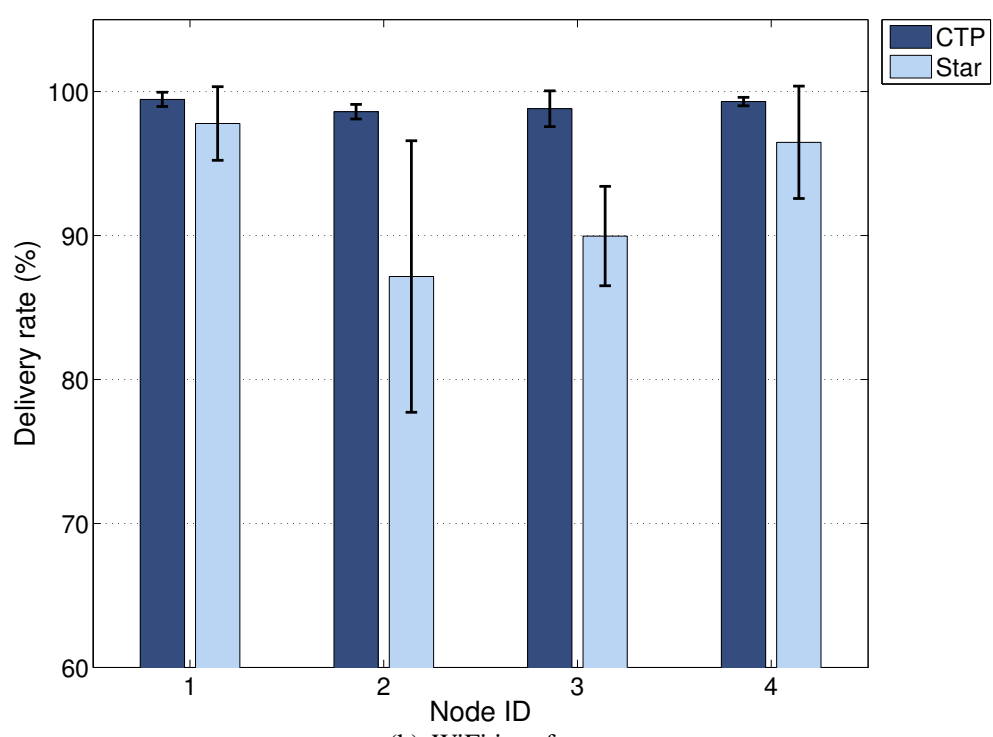

(b) WiFi interference

Fig. 17. Delivery rate of CTP vs. the star protocol (a) under Passengers-move-in-and-out scenario (b) with WiFi interferences, Tx power - 10 dBm and generation rate $10 \mathrm{pkts} / \mathrm{sec}$ per node. The error bars show one standard deviation from the mean over 5 six-minutes experiments. 


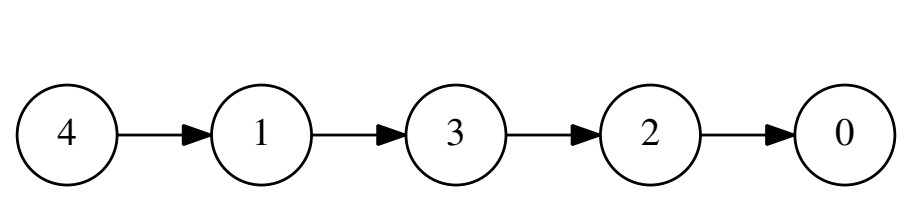

(a) Passengers-move-in-and-out scenario

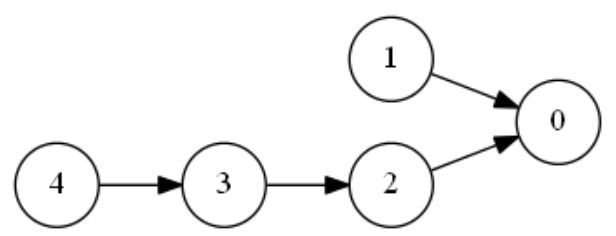

(b) WiFi interference

Fig. 18. Multi-hop CTP topology under (a) passengers-move-in-and-out scenario (b) with WiFi interference. 

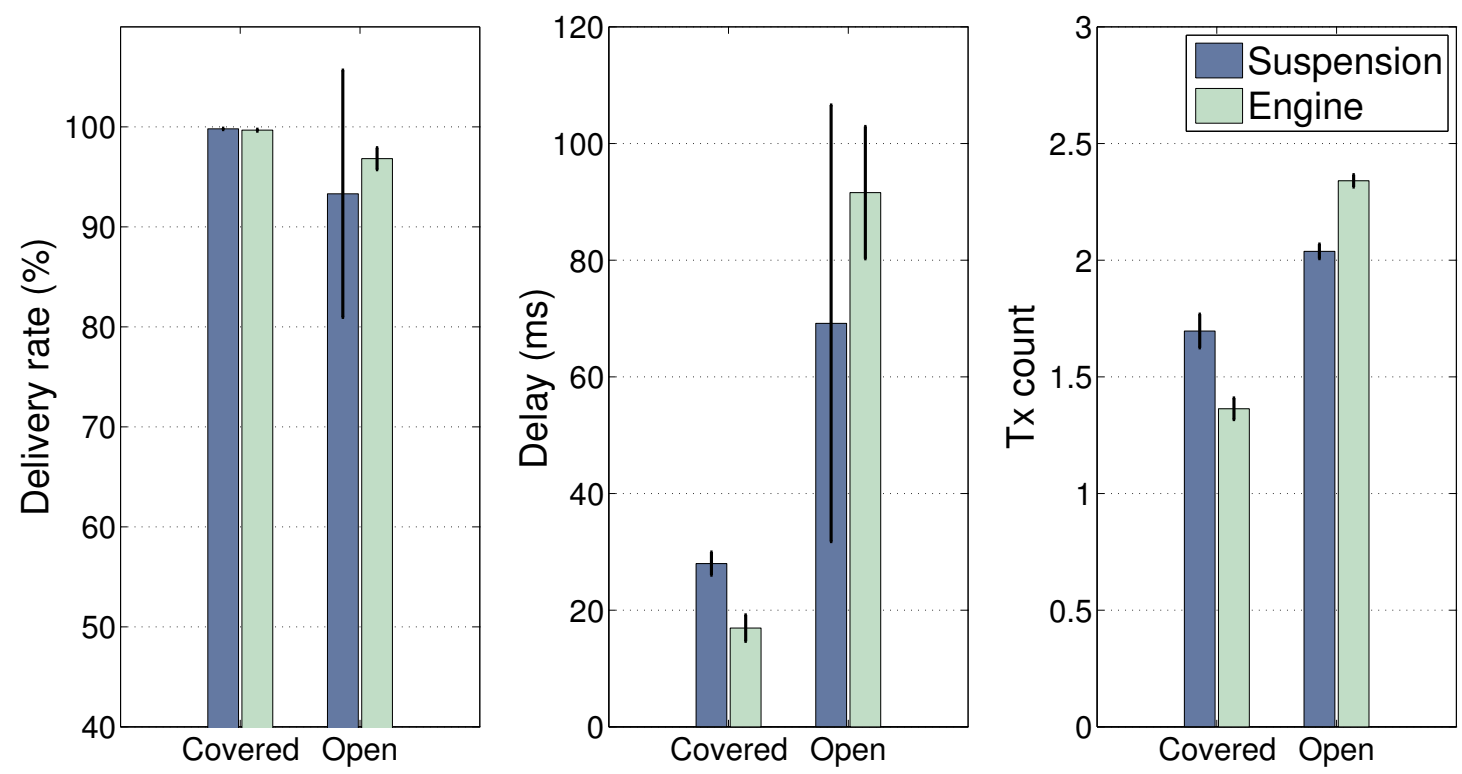

Fig. 19. CTP performance of the suspension network and the engine network in a covered and an open area parking with Tx power $-10 \mathrm{dBm}$ and the packet generation rate $10 \mathrm{pkts} / \mathrm{sec}$ per node. 

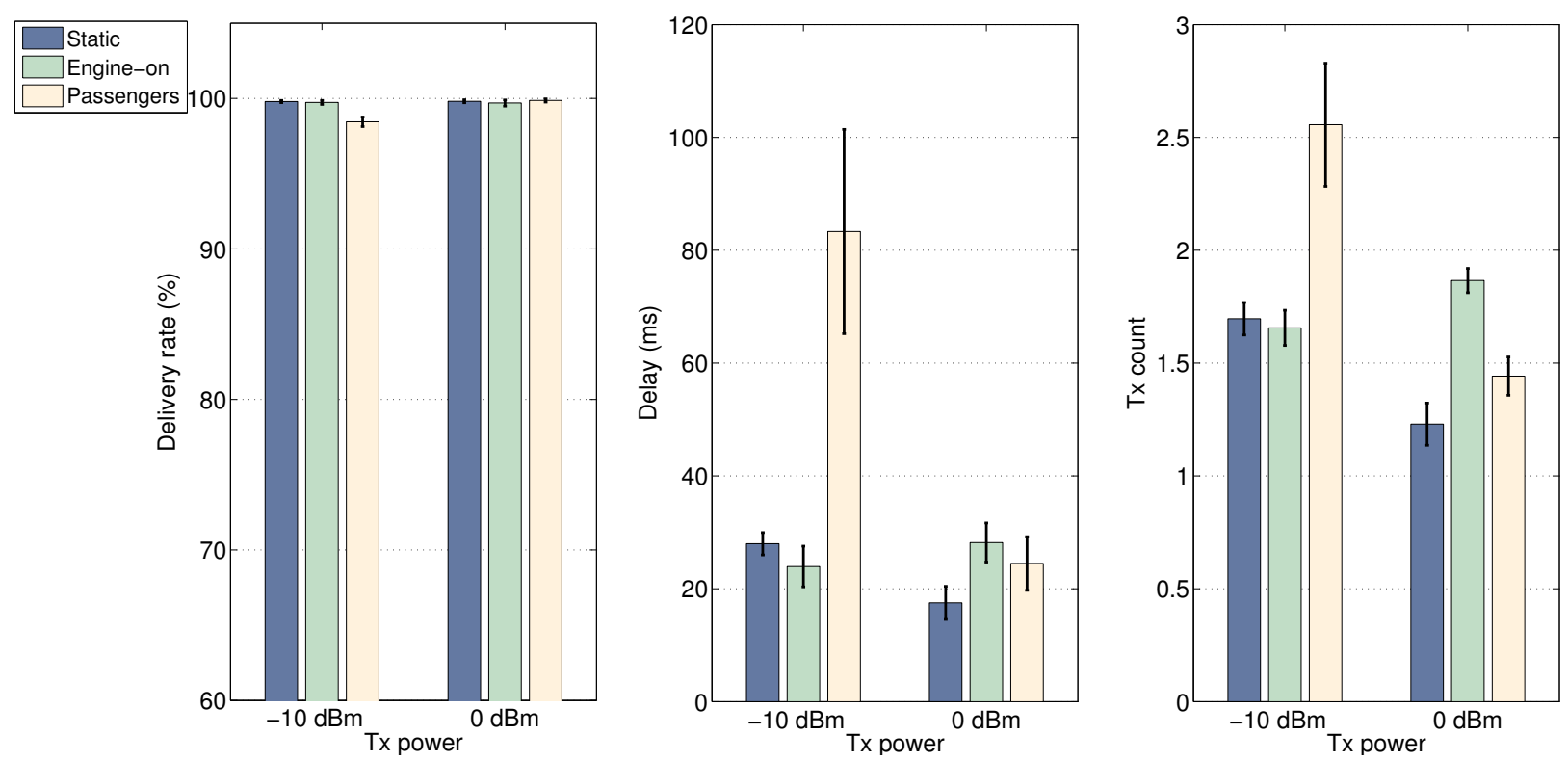

Fig. 20. CTP performance under engine-on and passengers-move-in-and-out conditions for the suspension network and the packet generation rate 10 pkts/sec per node. 

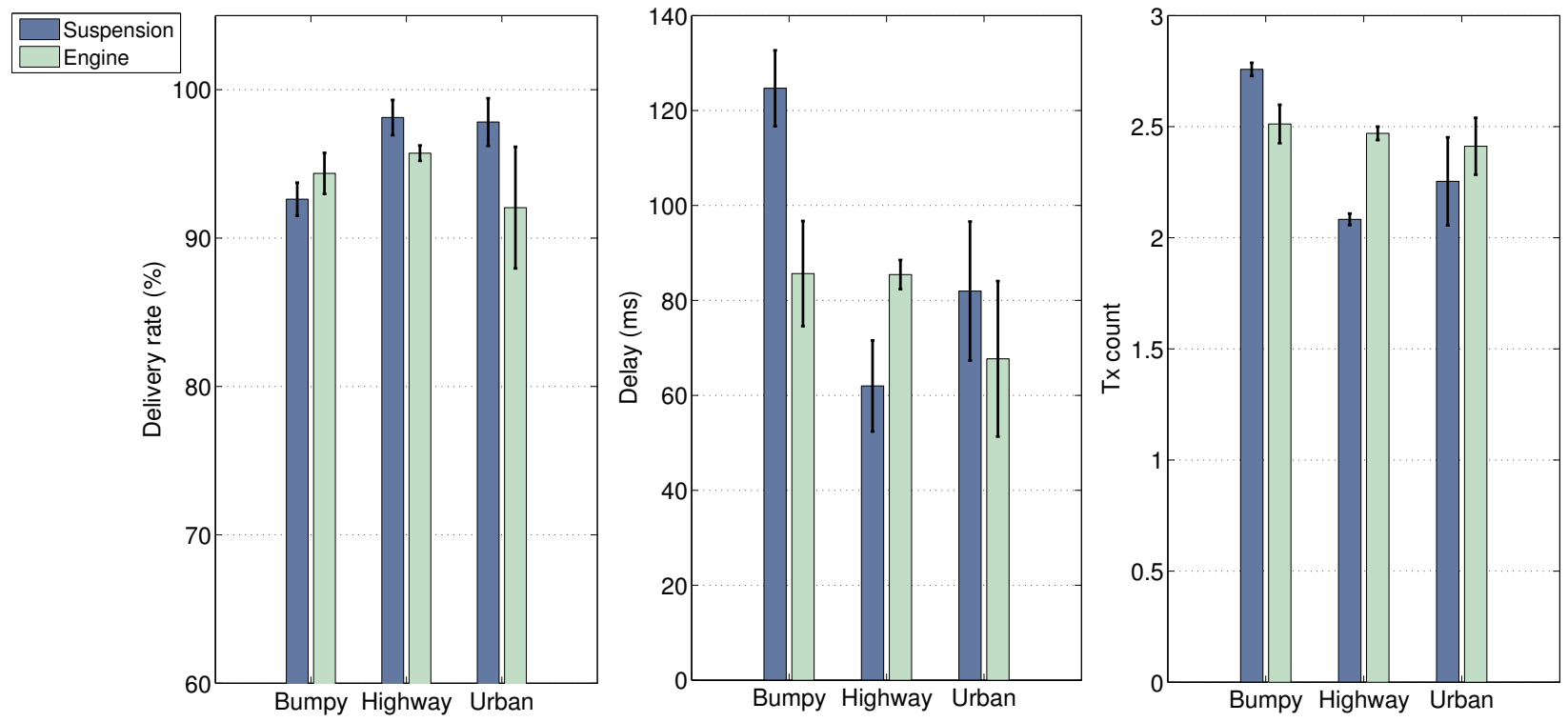

Fig. 21. CTP performance of the suspension network and the engine network within various driving conditions. Tx power is set to $-10 \mathrm{dBm}$ and the packet generation rate is $10 \mathrm{pkts} / \mathrm{sec}$ per node. 

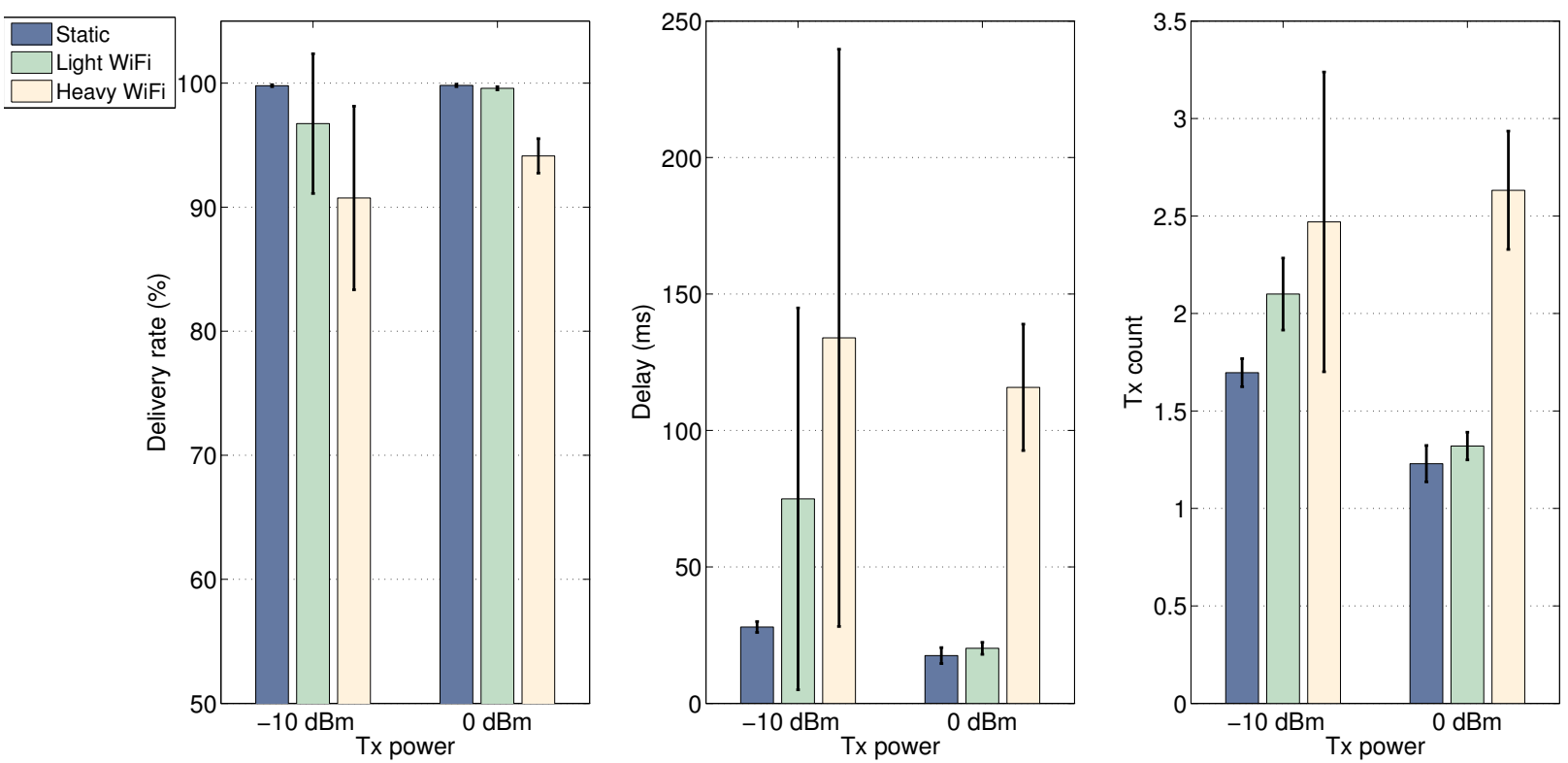

(a) Performance of the suspension network under WiFi interference
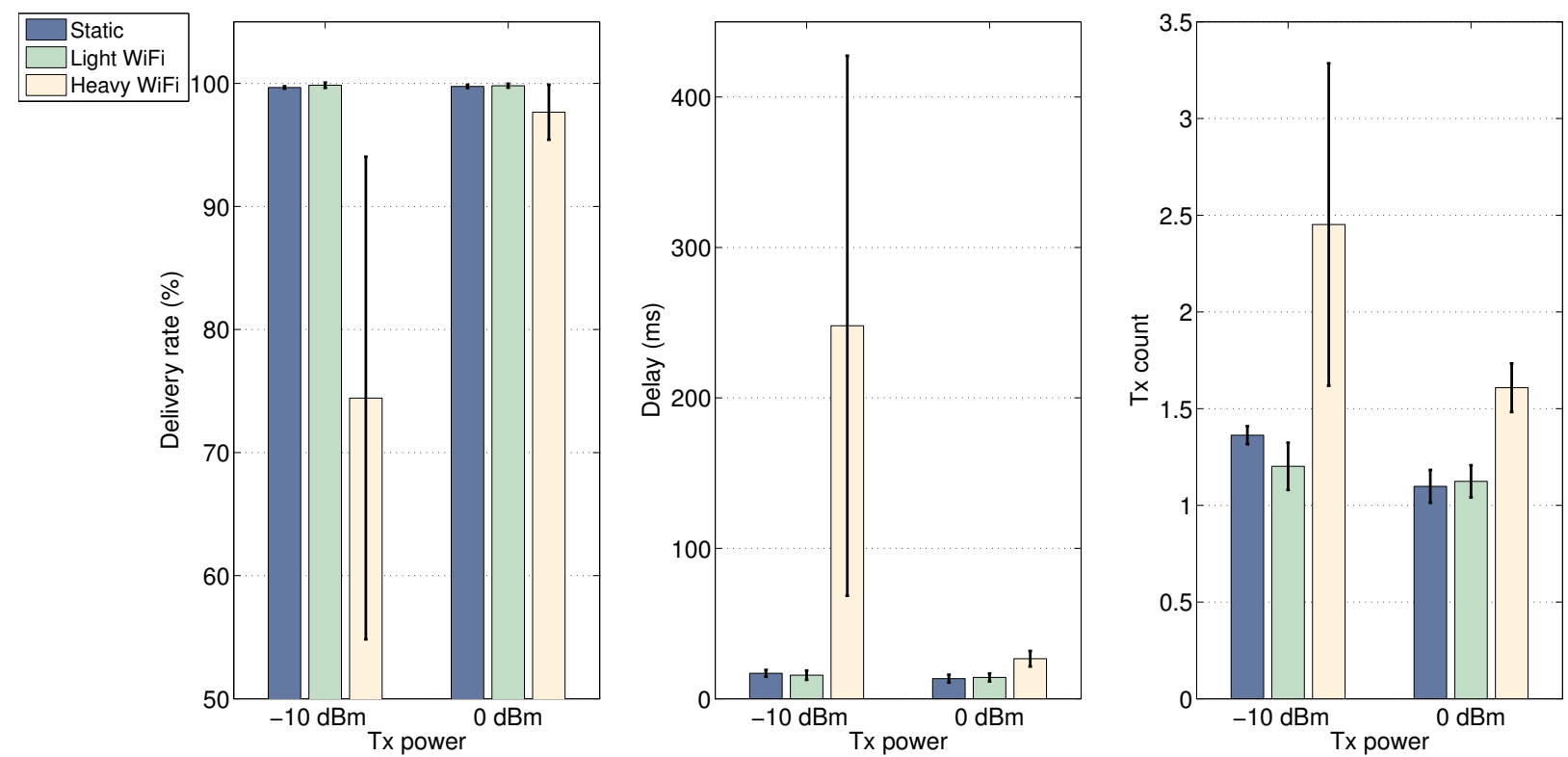

(b) Performance of the engine network under WiFi interference

Fig. 22. CTP performance under external WiFi interference for (a) the suspension network and (b) the engine network with the packet generation rate 10 pkts/sec per node. 